\title{
A modification of the arcsine-log calibration curve for analysing soil test value-relative yield relationships
}

Article in Crop and Pasture Science · March 2017

DOI: $10.1071 /$ CP16444

CITATIONS

2

4 authors:

\section{Adrián Alejandro Correndo}

International Plant Nutrition Institute

19 PUBLICATIONS 10 CITATIONS

SEE PROFILE

\section{Fernando Garcia}

International Plant Nutrition Institute, Argentina 93 PUBLICATIONS 1,124 CITATIONS

SEE PROFILE
READS

249

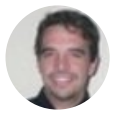

Fernando Salvagiotti

Instituto Nacional de Tecnología Agropecuaria 38 PUBLICATIONS 677 CITATIONS

SEE PROFILE

Flavio Hernan Gutierrez Boem

University of Buenos Aires

42 PUBLICATIONS 549 CITATIONS

SEE PROFILE

Some of the authors of this publication are also working on these related projects: 


\section{A modification of the arcsine-log calibration curve for analysing soil test}

\section{value - relative yield relationships*}

Adrián A. Correndo ${ }^{A, D}$, Fernando Salvagiotti ${ }^{B}$, Fernando O. Garcia ${ }^{A}$ and Flavio H. Gutiérrez-Boem ${ }^{C}$

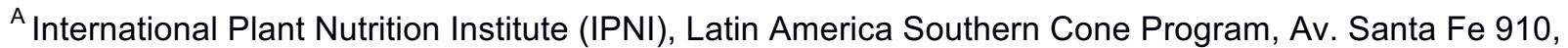
Acassuso, Buenos Aires Argentina.

${ }^{B}$ Dep. Agronomía, EEA INTA Oliveros, Santa Fe, Argentina.

C University of Buenos Aires, College of Agronomy, INBA-CONICET, Buenos Aires, Argentina.

${ }^{D}$ Corresponding author. Email: correndo@agro.uba.ar

*Pre-typeset proof version of: Correndo, A.A., F. Salvagiotti, F.O. García, and F.H. Gutiérrez Boem. 2017. A modification of the arcsine-log calibration curve for analysing soil test value-relative yield relationships. Crop \& Pasture Science 68 (3): 297-304. https://doi.org/10.1071/CP16444

Abstract. This article aims to discuss the arcsine-log calibration curve (ALCC) method designed for the Better Fertiliser Decisions for Cropping Systems (BFDC) to calibrate relationships between relative yield (RY) and soil test value (STV). Its main advantage is estimating confidence limits of the critical value (CSTV). Nevertheless, intervals for 95\% confidence level are often too wide, and authors suggest to reduce the confidence level to $70 \%$ in order to achieve narrower estimates. Still, this method can be further improved by modifying specific procedures. For this purpose, several datasets belonging to the BFDC were used. For any confidence level, estimates with the modified-ALCC procedures were always more accurate as compared with the original-ALCC. The overestimation of confidence limits with the original-ALCC was inversely related to the correlation coefficient of the dataset, which might allow a relatively simple and reliable correction of previous estimates. In addition, since the method is based on the correlation between STV and RY, the importance to test it for significance it is emphasized in order to support the hypothesis of a relationship. Then, the modified-ALCC approach could also allow a more reliable comparison of datasets by slopes of the bivariate linear relationship between transformed variables.

Additional keywords: bivariate model; correlation; standardized major axis regression. 


\section{Introduction}

32 When developing fertilizer recommendation models based on STV, the most usual goal is to

33 identify a critical value or range of a soil fertility variable for a given level of crop yield under

34 which response to fertilization is most likely. The most common approach is to fit a regression

35 line between crop yield and STV, the latter as the independent variable and the crop yield,

36 many times expressed as RY, as the dependent one. Mathematical functions used to describe

37 this relationship may be linear-plateau, quadratic-plateau or exponential (Mistcherlich) among

38 others (Mallarino and Blackmer, 1992; Colwell, 1963).

40 The most widely method used for fitting regression models, the ordinary least squares (LS)

41 approach, assumes that only the dependent variable (e.g. RY) is random while the explanatory

42 or regressor variable (e.g. STV) is considered as fixed and error-free. This approach is

43 especially valid for cases in which the explanatory variable is truly fixed, such as fertiliser rate.

44 However, when this variable is not controlled by the researcher, as happens with STV,

45 researchers normally still consider it as fixed. In this sense, it has been pointed out that LS

46 regression is frequently abused in soil research (Webster, 1997). When the underlying

47 relationship is bivariate it should be described as such and not as a predictive one. As well as

48 crop yield, the STV represents an "observed" dimension of the experiments, and comes from a

49 population that has a reference distribution and thus, an error component. Therefore, a joint

50 distribution of both variables called "bivariate" should be also considered, which its simplest

51 case is the "bivariate normal" (Legendre and Legendre, 1998).

53 Furthermore, calibrating RY vs STV often shows problems related to normality and

54 homogeneity of variance. This means a lack of statistical robustness of LS regression to

55 answer questions of interest (Kutner et al., 2005). Neither RY nor STV follow normal 
56 distribution, and thus, variable transformation is usually recommended (Webster, 2001).

57 However, the most common situation is transforming only the dependent variable (RY), while

58 keeping original units in the explanatory variable, and thus, the solution results partial. On the

59 other hand, if the nutrient under study is the only limitation to the crop growth, is expected that

60 high levels of STV may result in larger and also less variable yields each time. This behaviour

61 results in a dependence of RY variance on STV values when RY is the dependent variable.

62 Weighted regression is usually applied, but not always brings a solution (Motulsky and

63 Christopoulos, 2004).

64

65 On the other hand, an innovative approach has been recently proposed by Dyson and Conyers

66 (2013) for calibrating soil tests aimed at recommending crop fertilization. The ALCC (Arcsine

67 Logarithm Calibration Curve) method has been developed for determining CSTVs for nitrogen

$68(\mathrm{~N})$, phosphorus $(\mathrm{P})$, potassium $(\mathrm{K})$ and sulfur $(\mathrm{S})$ and response of various grain crops in

69 Australia (Anderson et al, 2013;. Bell et al, 2013a; 2013b; 2013c; Brennan and Bell, 2013). As

70 opposed to most commonly used calibrating methodologies, the ALCC method: (i) transforms

71 both variables involved in the relationship (i.e. RY and STV), and (ii) reverses the axes (i.e. fit

72 STV vs. RY) to estimate not only the CSTV for a given RY level but also its confidence interval $73(\mathrm{Cl})$.

75 In the original study (Dyson and Conyers, 2013), authors highlighted that estimations of $\mathrm{Cl}$ in 76 the original methodology were often too wide for making reliable recommendations and 77 comparisons between datasets. Therefore, they suggested to reduce the confidence level from $7895 \%(P=0.05)$ to $70 \%(P=0.30)$ in some comparisons (Dyson and Conyers, 2013; Watmuff et 79 al., 2013). However, a detailed review of the original ALCC method suggests that it is possible 
80 to achieve more accurate estimates of CSTVs without reducing the level of confidence by

81 modifying specific procedures.

82

83 The objectives of this study were to: (i) evaluate changes in procedures of the ALCC method in 84 order to obtain CSTV with narrower Cl; (ii) test the reliability of the shape of STV:RY 85 relationships based on a simple linear parameter, and (iii) discuss the importance of testing the 86 correlation coefficient for significance in order to support the hypothesis of a relationship 87 between variables.

\section{Materials and Methods}

90 Data sources and analysis

91 Datasets were gathered from several sources:

92 Dataset \#1. The first dataset was obtained from the BFCD Interrogator Database (NSW DPI, 93 2012). It was intentionally the same as Dyson and Conyers (2013) described in their paper, 94 belonging to the National Soil Fertility Program (NSFP) from 1968-72 (Fig. 1). The follow filters 95 in the BFDC Interrogator were applied to obtain this dataset: Nutrient = 'P', Farming System = 96 'dryland', From Year = '1968', To Year = '1972', State = 'Victoria', Season = 'winter', Crop = 97 'cereal wheat', Australian Soil Class = 'All', Soil Test and sample depth = 'P Colwell mg/kg at 0$9810 \mathrm{~cm}$ ', Trial quality = 'A trials only'.

\section{FIG 1}

102 Dataset \#2. A second dataset was also defined using the BFCD Interrogator Database in order

103 to make specific comparisons of parameters using the original and the modified ALCC method.

104 This dataset was obtained through the follow filters: Nutrient = 'P', Farming System = 'dryland', 
105 From Year $=$ 'All', To Year $=$ 'All', State $=$ 'All', Season $=$ 'winter', Crop $=$ 'cereal wheat',

106 Australian Soil Class $=$ 'Vertosol Black + Vertosol Grey', Soil Test and sample depth = 'P

107 Colwell $\mathrm{mg} / \mathrm{kg}$ at $0-10 \mathrm{~cm}$.

108

109 Dataset \#3. A third dataset was built for a comparison of standard errors (SE) of the CSTV

110 estimator. For this purpose, 60 datasets of STV and RY were used. They were gathered from

111 (i) the BFCD Interrogator Database (23) (NSW DPI, 2012), and (ii) published and unpublished

112 grain crop fertilisation experiments in the Pampean Region of Argentina (37) including several

113 crop-nutrient combinations (wheat, maize, and soybean crops, and N, P, S, and Zn). This

114 Dataset \#3 was defined using specific variables from each of the 60 datasets $(n=60)$ : (i)

115 correlation coefficient $\left(r_{x y}\right)$, (ii) SE of CSTV estimator using the original-ALLC method, and (iii)

116 SE of CSTV estimator using the modified-ALLC method.

118 All datasets were tabulated and processed in a Microsoft Excel $\AA$ environment in order to make

119 all comparisons. Analyses of obtained linear models were also checked in the R software

120 environment using packages Smatr v3.4-3 (Warton et al., 2012) and Stats v3.2.4 (R Core

121 Team, 2016). All figures were made with the GraphPad Prism software v7.0a for MacOSx

122 (GraphPad Software Inc., 2016).

\section{Procedures of the modified-ALCC}

125 A total of 9 steps are needed, each one can be performed with a common spreadsheet in

126 Microsoft Excel $\AA$ or similar. Essential commands for applying in a common spreadsheet are

127 included in parentheses. Note they could vary depending on the software version and

128 language. Steps \#1 to \#3 of the modified-ALCC are basically the same as the original-ALCC 
129 method (Dyson and Conyers, 2013). Specific differences are detailed in the Results and

130 Discussion section.

131 1. Transform variables. This will simplify the relationship between variables as a simple 132 linear equation. Transformations are:

133 a. Natural logarithm for the STV, hereinafter $Y\left(=L N\left(S T V_{i}\right)\right)$. The method does work independently of STV units, which normally are expressed in $\mathrm{kg} / \mathrm{ha}$, $\mathrm{mg} / \mathrm{kg}, \mathrm{cmol}_{\mathrm{d}} / \mathrm{kg}$, among others.

b. Arcsine of the square root for the RY, hereinafter $X\left(=A \operatorname{SIN}\left(\operatorname{SQRT}\left(R Y_{i} / 100\right)\right)\right)$. The original RY units must be always expressed with respect to a maximum yield (observed or estimated), see Dyson \& Conyers (2013) for additional details.

2. Center the $X$ variable, with respect to the RY level for which you want to estimate the CSTV (=ASIN(SQRT(RY $\left./ 100))-A S I N\left(S Q R T\left(R Y_{\text {goal }} / 100\right)\right)\right)$. For example, for a $R Y_{\text {goal }}=90 \%$ we need to subtract, from each value of $X$, the corresponding $\operatorname{arcsine} \sqrt{ }(90 / 100)=1.249$

3. Estimate the Pearson correlation coefficient $\left(r_{x y}\right)$, between $X$ (centered) and $Y$ (=PEARSON $\left(\mathrm{X}_{\text {values }}, \mathrm{Y}_{\text {values }}\right)$. Since the methodology is based on "correlation" between variables, it is advisable to test this coefficient for significance before the next steps.

4. Estimate the average means of centered $X$ (=AVERAGE $\left.\left(X_{\text {values }}\right)\right)$ and $Y$ (=AVERAGE $\left.\left(\mathrm{Y}_{\text {values }}\right)\right)$. They represent coordinates of the data ellipse centroid $(\bar{X}, \bar{Y})$, where all possible regressions pass through.

150 5. Estimate a linear regression (Eqn. 1) between $X$ and $Y$ values (Fig. 2) using the ordinary least squares (LS) approach. 
Where, $\widehat{Y}_{i L S}$ are the fitted LS values of $\operatorname{In}(\mathrm{STV})$ and $X_{i}$ are the observed (and centered) $X$ values (see Step \#2).

6. Estimate the bivariate equation between $\mathrm{X}$ and $\mathrm{Y}$. This step basically consists in rotating the LS regression (Eqn. 1) about the centroid of the data ellipse (Step \#4). The equation of interest is called standardized major axis (SMA), which describes a structural or bivariate relationship between variables based on correlation. There is specific software for fitting this type of regression (Warton et al., 2012). However, the most direct and simplest way is to use a mathematical property that relates slopes of LS and SMA regressions (Eqn. 2) (Legendre and Legendre, 1998). Thus, since all possible regressions of any data ellipse pass through the centroid coordinates $(\bar{X}, \bar{Y})$ (Eqn. 3), we can estimate the SMA intercept $\left(\hat{\alpha}_{S M A}\right)$ by Eqn. 4. Finally, we obtain the complete SMA equation, which for the example application is shown in Fig. 2B. Note that Eqn. 2 is not plausible when $r_{x y}=0$, so correlation is recommended to be tested for significance first (see "Testing correlation significance" section). 
176 7. Estimate the CSTV. It has to be consider the model when $X=0$. As in this example, the $177 X$ values are centered on $\mathrm{RY}=90 \%$, the intercept represents the CSTV $_{90 \%}$. Since the 178 estimator $\left(\hat{\alpha}_{S M A}\right)$ is expressed in logarithmic units (Eqn. 5), it is necessary to back179 transform it to its original units (Eqn. 6, =EXP $\left.\left(\hat{\alpha}_{S M A}\right)\right)$.

180

$\hat{\alpha}_{S M A}=\ln \left(C S T V_{90}\right)$

182

185 8. Estimate the confidence interval $(\mathrm{Cl})$ of the CSTV. To estimate the confidence limits of the CSTV we must use Eqn. 7 and Eqn.8 which have been described as the most appropriate to estimate the $\mathrm{Cl}$ of intercept for SMA regression (Warton et al., 2006). Therefore, a $\mathrm{Cl}$ with $95 \%$ of confidence level equals approximately (depending on the sample size $n) \pm 2$ standard errors (SE), while a $\mathrm{Cl}$ with $70 \%$ of confidence level equals approximately \pm 1 SE.

$S E \hat{\alpha}_{S M A}=\sqrt{\frac{\sum_{i=1}^{n}\left(y_{i}-\hat{y}_{i S M A}\right)^{2}}{n-2} *\left[\frac{1}{n}+\frac{\bar{x}^{2}}{\sum_{i=1}^{n}\left(x_{i}-\bar{x}\right)^{2}}\right]}=\sqrt{M S E *\left[\frac{1}{n}+\frac{\bar{x}^{2}}{S S_{x}}\right]}$ Where, $S E \hat{\alpha}_{S M A}$ represents the standard error of the intercept, $y_{i}$ are the observed $Y$ values, $\hat{y}_{i S M A}$ are the fitted SMA values $\left(=\hat{\alpha}_{S M A}+\hat{\beta}_{S M A} * X_{i}\right), n$ is the sample size, $n-2$ are degrees of freedom (df), MSE represents the mean square error of the model $\left(=\left(\operatorname{SUM}\left(\left(y_{i}-\right.\right.\right.\right.$ 
Where, $S E \hat{\alpha}_{S M A}$ represents the standard error of the intercept (Eqn. 7), and $t$ is the two-tailed Student- $t_{\frac{\alpha}{2}}$ value for an $\alpha$ significance level and $n-2 \mathrm{df}(=\operatorname{TINV}(\alpha, \mathrm{df}))$.

9. Draw the curve. To fit a RY vs STV curve, we must solve the equation based on the ALCC method. The ALCC curve does not describe a causal relationship (predictive) but a bivariate relationship (back-transformed) instead. Fitted values of $\ln ($ STV) are obtained by the SMA linear equation (Eqn. 9) and back-transformed STV values are obtained by Eqn. 10. Finally, for the same range of fitted values with Eqn. 9, we can also express the RY values (\%) using the parameters of the bivariate relationship (Eqn.

$$
\hat{Y}_{i S M A}, \ln (S T V)=\hat{\alpha}_{S M A}+\hat{\beta}_{S M A} *\left[\operatorname{arcsine} \sqrt{\frac{R Y}{100}}-\operatorname{arcsine} \sqrt{\frac{R Y \text { goal }}{100}}\right]
$$

$$
R Y(\%)=100 *\left\{\sin \left[\operatorname{arcsine}\left(\sqrt{\frac{R Y_{\text {goal }}}{100}}\right)+\frac{\hat{P}_{i S M A}-\widehat{\alpha}_{S M A}}{\widehat{\beta}_{S M A}}\right]\right\}^{2}
$$

\section{Results and Discussion}

\section{Confidence intervals of CSTVs}

218 Since both variables are inexact, Dyson and Conyers (2013) make focus in finding a 'major

219 axis equation' of the data ellipse. However, the way they reach it has an impact on the error 220 size of the model. In order to get the major axis equation (in this case a standardized major 
221 axis), they apply a second transformation of the already transformed $\ln (\mathrm{STV})$. This step is 222 designated as the "r-modification procedure", but the specific equation used for this second 223 transformation is not properly described in the paper. The equation is described in the Eqn. 12 224 (Dyson, pers. comm.). This second transformation produces a new variable $\left(Y_{r-\text { modified }}\right)$ which 225 has a wider range of values than the original one $(Y)$. Then by a LS regression of $Y_{r \text {-modified }}$ 226 values on $\mathrm{X}$ (Eqn. 13), they reach the structural relationship of interest. Even though the mean

227 values of intercept $\left(\hat{\alpha}_{L S r}\right)$ and slope $\left(\hat{\beta}_{L S r}\right)$ parameters are correct, the "r-modification 228 procedure" generates an unnecessary error overestimation of the model, and thus affects the 229 precision when estimating the CI of CSTV.

$$
Y_{i-r \text { modified }}=\bar{Y}+\left(\frac{Y_{i}-\bar{Y}}{r_{x y}}\right)
$$

$$
\hat{Y}_{i L S r}=\hat{\alpha}_{L S r}+\hat{\beta}_{L S r} * X_{i}
$$

235 Where, $\hat{Y}_{i L S r}$ are the fitted r-modified values of $\ln (\mathrm{STV})$ and $X_{i}$ are the observed $\mathrm{X}$ values (centered).

237 In cases with wide $\mathrm{Cl}_{95 \%}$ for CSTV, Dyson and Conyers (2013) suggests to also estimate the 238 CSTV with a lower confidence level $\left(\mathrm{Cl}_{70 \%}\right)$ in order to achieve narrower estimates, especially 239 for the BFDC Interrogator (Conyers et al., 2013; Watmuff et al., 2013). However, this issue of 240 wide $\mathrm{Cl}$ of CSTVs lies on the r-modification procedure which generates a "wider in $\mathrm{Y}$ " data

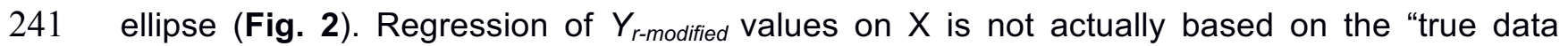
242 ellipse", where the bivariate major axis equations are based on (Jolicoeur, 1990; Sokal and 243 Rohlf, 1995; Warton et al., 2006). In this sense, we suggest to modify this procedure to obtain 
244 the bivariate relationship of interest between transformed variables without the error 245 overestimation.

247 Instead of using the $r$-modification of $Y$ values, we propose to use a bivariate approach called 248 'standardized major axis regression (SMA)'. This approach is not a prediction of $\mathrm{Y}$ depending

249 on $\mathrm{X}$ as usual. It is based on representing in one dimension -or axis- data that varies in two 250 dimensions, which could be called as a bivariate relationship (Warton et al., 2006). The model 251 assumptions are the same as usual: independency, normal distribution of error and 252 homoscedasticity. Transformation seems to play an important role for fulfilling the last two 253 assumptions, which is exemplified for Dataset \#1 (Fig. 3). In addition, correlation between 254 variables and whether data follow a distribution that approximates a bivariate normal or not 255 should be checked out.

257 In the particular case of interest (describing a relationship between RY and STV), there are 258 three main characteristics that determine the usefulness of the SMA approach: (i) RY and STV 259 represent two observed variables or dimensions of the same experiments, (ii) standardization 260 allows using variables that do not have comparable scales of measure, and (iii) the 261 independence from any causal relationship between variables, then the $\mathrm{X}: \mathrm{Y}$ direction of SMA 262 regression is functional to the objectives of the researcher.

\section{FIG 3.}

266 With the SMA regression approach, we can estimate exactly the same equation as the original-

267 ALCC algorithm but avoiding the $\mathrm{Cl}$ overestimation of the intercept parameter $(\hat{\alpha})$, which is the 268 CSTV estimator (Fig. 2). Consequently, keeping the same level of confidence (e.g. 95\%), the 
$269 \mathrm{Cls}$ of the modified-ALCC algorithm are always more accurate than the original-ALCC. For the

270 Dataset \#1, the SMA equation shows more accurate estimates of the intercept $\left(\hat{\alpha}_{S M A}\right.$,

$\left.271 \mathrm{Cl}_{95 \%}=2.963-3.198\right)$ as compared with the LS regression of $\mathrm{Y}_{\mathrm{r} \text {-modified }}$ values used by the original-

272 ALCC method $\left(\hat{\alpha}_{L S_{r}} ; \mathrm{Cl}_{95 \%}=2.819-3.341\right)$. These results are also observed for Dataset \#2, with

273 CSTV estimaties from $+30.3 \%$ to $+61.4 \%$ more accurate for the modified-ALCC as compared

274 to the original-ALCC algorithm (Table 1).

275

276

\section{TABLE 1}

277

278

Based on a 60 datasets comparison (Dataset \#3), we also observed that the overestimation of

the SE $\hat{\alpha}$ (Eqn. 14) was inversely proportional to the correlation coefficient $\left(r_{x y}\right)$ of a dataset

280 (Fig. 4). For the analysed cases $(n=60)$, the overestimation varied from $+10.6 \%$ to $+222.5 \%$ for

281 datasets with $r_{x y}$ of 0.245 and 0.875 , respectively. This inverse relationship is explained by the

$282 r$-modification procedure which retransforms the $\ln (\mathrm{STV})$ values based on the $r_{\mathrm{xy}}$ coefficient

283 (Eqn. 12). However, the relationship described in Fig. 4 might also allow a relatively simple

284 and reliable $\left(r^{2}=0.99\right)$ correction of previous estimations based on the original-ALCC algorithm

285 (e.g. for the BFDC Interrogator) just using the " $\mathrm{r}_{\mathrm{xy}}$ " coefficient of the dataset and Eqn. 8.

286

$$
S E \hat{\alpha}_{\text {overestimation }}(\%)=100 *\left(\frac{\text { SE } \widehat{\alpha}_{\text {original }}-\text { SE } \widehat{\alpha}_{\text {modified }}}{\text { SE } \widehat{\alpha}_{\text {modified }}}\right)
$$

\section{FIG 4}

289

290 Testing SMA slopes

291 Since the ALCC curve (back-transformed) comes from a bivariate linear relationship, the SMA

292 slope $\left(\hat{\beta}_{S M A}\right)$ can also be compared among datasets (Fig. 5A and 5B). It might be considered 
293 as an index of the ALCC curvature (Fig. 5C and 5D). Following Eqn. 10 -back-transformed in

294 terms of RY-, a greater $\hat{\beta}_{S M A}$ results in a less steep curve. In contrast, a smaller $\hat{\beta}_{S M A}$ results in 295 a steeper curve. This behaviour was observed in Dataset \#2 for wheat RY related to soil

296 Colwell-P level at 0-10 cm, where Vertosol Black soils showed a greater $\hat{\beta}_{S M A}$

$297\left(\hat{\beta}_{\text {Black-CI95\% }}=2.671-3.370\right)$ as compared to Vertosol Grey soils $\left(\hat{\beta}_{\text {Grey-CI95\% }}=1.610-2.333\right)$

298 which also means different fitted ALCC curve shapes (Fig. 5).

299

300

\section{FIG 5}

301

302 Dyson and Conyers (2013) proposed an estimation of the average slope (and its SE) from 50\% 303 to $80 \%$ of RY as the deficient zone of the curve. However, the formula is not specified for users 304 who want to apply the technique. Moreover, even if detailed, the comparison of slopes of SMA 305 regressions does not follow the same formula as the LS regression, as Dyson and Conyers (2013) followed after the r-modification procedure. In fact, as well as for intercept, the LS

307 regression of $Y_{r-\text { modified }}$ values also overestimates the error of the slope $\left(\hat{\beta}_{S M A}\right)$ as compared to

308 the true SMA approach (Fig. 2). For the Dataset \#1, the modified-ALCC approach showed a $30962.6 \%$ more accurate estimation for the slope $\left(\hat{\beta}_{S M A}, \mathrm{Cl}_{95 \%}=1.502-2.147\right)$ as compared with the 310 original-ALCC method $\left(\hat{\beta}_{L S_{r}}, \mathrm{Cl}_{95 \%}=0.933-2.659\right)$.

312 The SMA regressions have been used to study allometric relationships where the slope $\hat{\beta}_{S M A}$ is

313 the main parameter of interest (Warton et al., 2002). The confidence interval for the $\hat{\beta}_{S M A}$ can

314 be estimated at a predetermined confidence level, and checked whether a value of interest lies

315 inside or outside the confidence limits. The formula to compute $\mathrm{Cl}$ for SMA is different 316 compared to the LS regression (Eqn. 15) (Jolicoeur and Mosimann, 1968; Jolicoeur 1990; 
317 Sokal and Rohlf, 1995). A peculiarity of SMA regression is that the slope cannot be tested for

318 significance (Legendre and Legendre, 1998). This is a trivial case because $\hat{\beta}_{S M A}$ (Eqn. 2)

319 cannot be zero unless the standard deviation of $Y\left(s_{y}\right)$ is equal to zero. For this reason, among

320 others, the importance of testing the correlation for significance is discussed below.

321

$322 C I_{\widehat{\beta}_{S M A}}=\hat{\beta}_{S M A} *(\sqrt{(\beta+1)} \pm \sqrt{\beta})$, where $\beta=\frac{t^{2} * 1-r_{x y}^{2}}{n-2}$

323 Where, $\hat{\beta}_{S M A}$ represents the slope value, $r_{x y}$ is the correlation coefficient of dataset, $t$ represents a two-

324 tailed Student's $t_{\frac{\alpha}{2}}$ value for an $\alpha$ significance level, and $n-2$ are degrees of freedom.

325

326 Testing correlation significance

327 A criterion to exclude a dataset based only on its correlation strength was established by

328 Dyson and Conyers (2013). In the BFDC Interrogator, estimations will not be fitted if a dataset

329 has an $r_{x y}<0.2$. Despite this criterion is reasonably valid, it could not be enough for potential

330 users of the method. The significance of the correlation coefficient $r_{x y}$ should be tested first in

331 order to determine if a relationship between variables is supported (McArdle, 1988). A

332 relationship could be weak but significant or could be strong and yet not significant, where the

333 sample size $(n)$ might play a key role. For large sample sizes it is easy to achieve significance

334 and one should also consider the strength of correlation to determine whether the relationship

335 explains very much or not. Conversely, for small sample sizes it could be easy to produce a

336 strong correlation by chance and one should also consider its significance to keep from

337 rejecting a true null hypothesis. Additionally, as discussed above, the SMA slope is only

338 meaningful when $r_{x y}$ is different from zero (Eqn. 2). Therefore, it is advisable to evaluate not

339 only the correlation strength but also its significance for a better interpretation of data. Since

340 correlation between STV and RY is normally expected be positive $\left(r_{x y}>0\right)$, the commands to 
341 test it in a spreadsheet is $=\operatorname{TDIST}\left(t_{r}, d f, 1\right)$, where $t_{r}$ is the t-statistic (Eqn. 16) and $d f$ are

342 degrees of freedom.

$343 \quad t_{r}=\frac{r_{x y} * \sqrt{n-2}}{\sqrt{r_{x y}^{2}}}$

345 Conclusions

346 The ALCC algorithm is an interesting approach for estimating CSTVs, which cope with

347 problems usually faced when using traditional regression methods for calibrating soil tests data

348 (i.e. lack of normality and homoscedasticity, both variables measured with error). The modified-

349 ALCC method described in this paper, even when it requires some additional steps (and

350 probably add complexity), it also incorporates comparative advantages over the original-ALCC

351 method. Based on the SMA regression, it produces more accurate estimates of CSTVs and

352 their confidence intervals, as well as more reliable comparisons between datasets.

353

354 Acknowledgements

355 Authors want to give special thanks to Dr. CB Dyson (South Australian Research and

356 Development Institute) and Dr. MK Conyers (NSW Department of Primary Industries) for their

357 willingness to make clarifications about the original-ALCC methodology. We also thank to Dr.

358 ET Peltzer (MBA Research Institute) for his selfless advice about bivariate methods.

360 References

361 Anderson, GC, Peverill KI, Brennan RF (2013) Soil sulfur-crop response calibration relationships and

362 criteria for field crops grown in Australia. Crop and Pasture Science 64:523-530.

363 http://dx.doi.org/10.1071/CP13244 
364 Bell MJ, Moody PW, Anderson GC, Strong W (2013)a. Soil phosphorus-crop response calibration 365 relationships and criteria for oilseeds, grain legumes and summer cereal crops grown in Australia. Crop

366 and Pasture Science 64:499-513. http://dx.doi.org/10.1071/CP12428

367 Bell MJ, Strong W, Elliott D, Walker C (2013)b. Soil nitrogen—crop response calibration relationships

368 and criteria for winter cereal crops grown in Australia. Crop and Pasture Science 64:442-460.

369 http://dx.doi.org/10.1071/CP12431

370 Bell R, Reuter D, Scott B, Sparrow L., Strong W., Chen W (2013)c. Soil phosphorus-crop response

371 calibration relationships and criteria for winter cereal crops grown in Australia. Crop and Pasture

372 Science 64:480-498. http://dx.doi.org/10.1071/CP13016

373 Brennan RF, Bell MJ (2013) Soil potassium—crop response calibration relationships and criteria for field

374 crops grown in Australia. Crop \& Pasture Science 64:514-522. http://dx.doi.org/10.1071/CP13006

375 Colwell JD (1963) The estimation of phosphorus fertilizer requirements of wheat in Southern New South

376 Wales by soil analysis. Australian Journal of Experimental Agriculture and Animal Husbandry 3, 190377197.

378 D'Agostino RB, Belanger A, D'Agostino Jr RB (1990) A suggestion for using powerful and informative

379 tests of normality. The American Statistician 44(4):316-321. http://dx.doi.org/10.2307/2684359

380 Dyson CB, Conyers MK (2013) Methodology for online biometric analysis of soil test-crop response

381 datasets. Crop \& Pasture Science 64, 435-441. http://dx.doi.org/10.1071/CP13009

382 GraphPad Software Inc. (2016) GraphPad Prism v7.0a for MacOSX, La Jolla, CA, USA.

383 http://www.graphpad.com/guides/prism/7/user-guide/index.htm

384 Jolicoeur P (1990) Bivariate allometry: interval estimation of the slope of the ordinary and standardized

385 normal major axes and structural relationship. Journal of Theoretical Biology 144, 275-285.

386 Jolicoeur P, Mosimann JE (1968) Intervalles de confiance pour la pente de l'axe majeur d'une 387 distribution normale bidimensionnelle. Biometrie-Praximetrie 9: 121-140.

388 Kutner MH, Nachtsheim CJ, Neter J, Li W (2005) Applied Linear Statistical Models. (5th ed.). New York:

389 McGraw-Hill. 1396pp. ISBN 0-07-238688-6

390 Legendre P, Legendre L (1998) Numerical ecology. Numerical Ecology Second English Edition, 20(20), $391870 p$. 
392 Mallarino AP, Blackmer AM (1992) Comparison of methods for determining critical concentrations of soil

393 test phosphorus for corn. Agron J, 84, 850:856.

394 McArdle B (1988) The structural relationship: regression in biology. Can. J. Zool., 66: 2329-2339.

395 Motulsky H, Christopoulos A (2004) Fitting Models to Biological Data using Linear and Nonlinear

396 Regression. A Practical Guide to Curve Fitting, Oxford University Press, NY.

397 NSW DPI (2012) Making Better Fertiliser Decisions for Cropping Systems in Australia: online database,

398 NSW DPI and the Grains Research and Development Corporation, last access date: Sep $4^{\text {th }} 2016$,

399 http://www.bfdc.com.au

400 R Core Team (2016) R: A language and environment for statistical computing. R Foundation for

401 Statistical Computing, Vienna, Austria. http://R-project.org

402 Speirs SD, Scott BJ, Moody PW, Mason SD (2013) Soil phosphorus tests II: A comparison of soil test-

403 crop response relationships for different soil tests and wheat. Crop \& Pasture Science 64, 469-479.

404 http://doi:10.1071/CP13111

405 Sokal RR, Rohlf FJ (1995) Biometry - The Principles and Practice of Statistics in Biological Research,

406 3rd Edn. W. H. Freeman, New York.

407 Warton DI, Weber NC (2002) Common slope tests for errors-in-variables models. Biometrical Journal 44, 408 161-174.

409 Warton DI, Duursma RA, Falster DS, Taskinen S (2012) Smatr 3 - an R package for estimation and

410 inference about allometric lines. Methods in Ecology and Evolution, 3(2): 257-259.

411 Warton DI, Wright IJ, Falster DS, Westoby M (2006) Bivariate line-fitting methods for allometry.

412 Biological Reviews of the Cambridge Philosophical Society, 81(2), $259-291$.

$413 \quad$ http://doi:10.1017/S1464793106007007

414 Watmuff G, Reuter DJ, Speirs SD (2013) Methodologies for assembling and interrogating N, P, K, and

415 soil test calibrations for Australian cereals, oilseed and pulse crops. Crop \& Pasture Science 64, 424416434.

417 Webster R (1997) Regression and functional relations. European Journal of Soil Science, September 418 1997, 48: 557-566. 
419 Webster R (2001) Statistics to support soil research and their presentation. European Journal of Soil 420 Science, June 2001, 52: 331-340. 


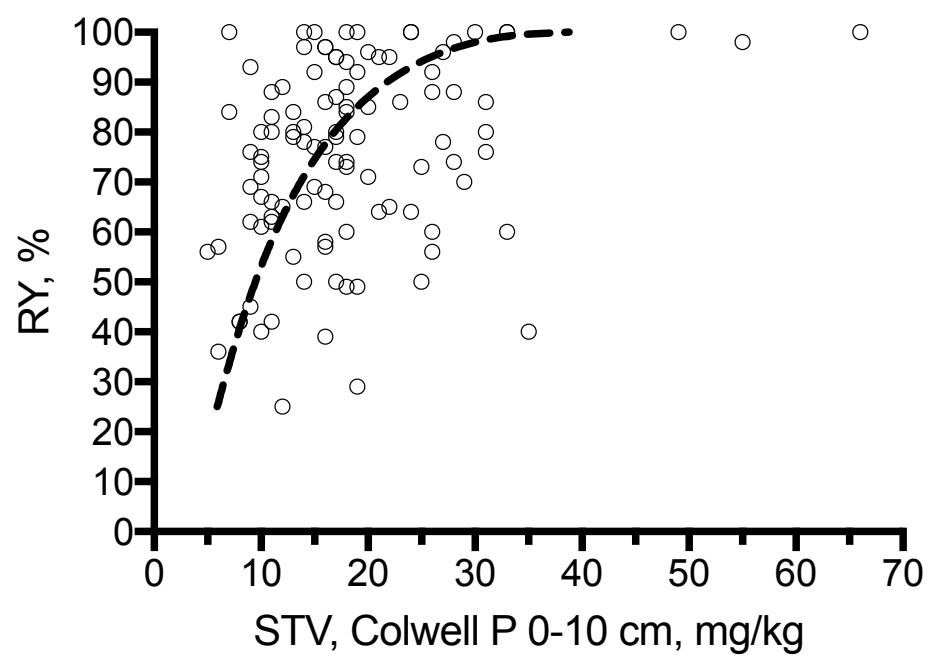

422

423 Fig. 1. Scatter plot of relative yield (RY, \%) and soil test value (STV, Colwell $P$ at $0-10 \mathrm{~cm}, \mathrm{mg} / \mathrm{kg}$ ) for

424 Dataset \#1 -107 experiments from the National Soil Fertility Program (NSFP, 1968-72) in Victoria (107

425 class A trials)-. Data was gathered from the BFDC Interrogator database following previous descriptions

426 given by Dyson and Conyers (2013). Dashed line represents the fitted calibration curve by the ALCC

427 approach (back-transformed from a linear regression between transformed variables -Fig. 2-). 


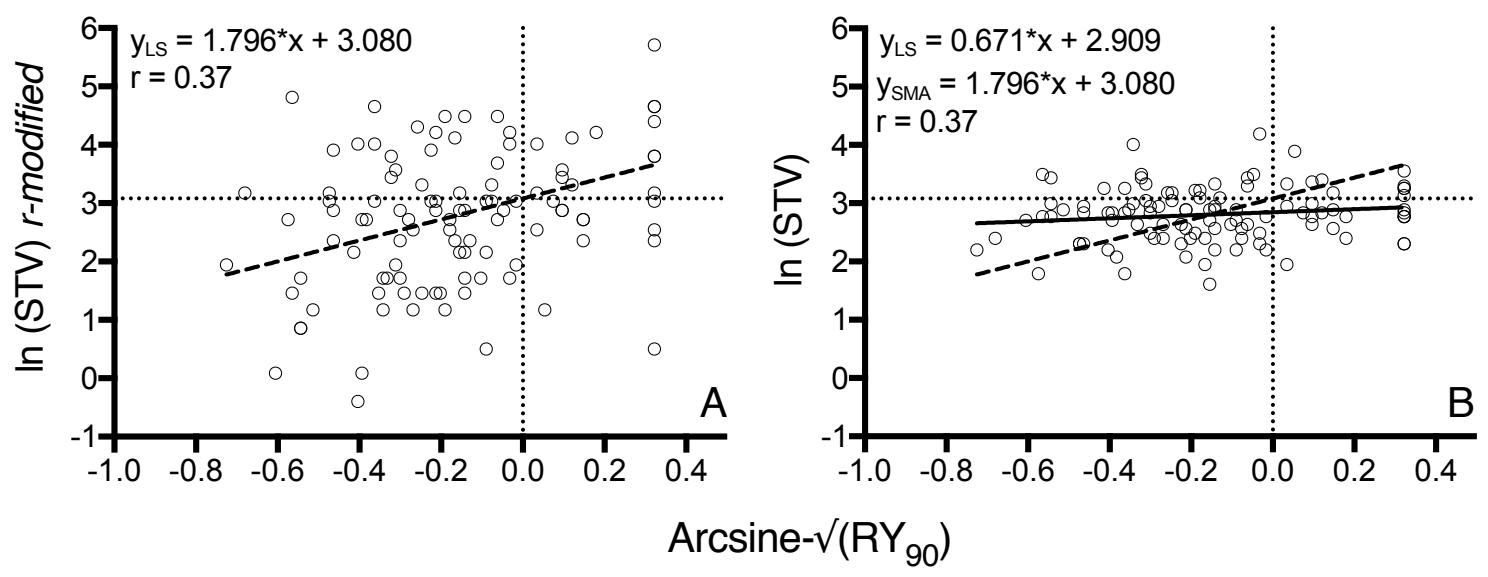

429

430 Fig. 2. Linear relationships between STV (Colwell $P, 0-10 \mathrm{~cm}$ ) and wheat relative yield (RY) both as 431 transformed variables of Dataset \#1. The same structural linear relationship (dotted lines) is derived from

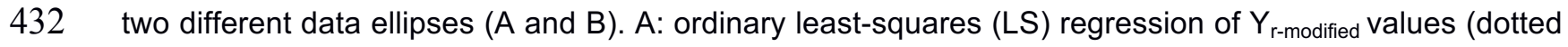
433 line) for the original ALCC (Dyson and Conyers, 2013). B: bivariate standardized major axis (SMA) 434 regression (dotted line) for the modified ALCC, derived from the LS regression of $\operatorname{In}(\mathrm{STV})-$ not $r$ 435 modified- on the arcsine of square root of centered RY. In both cases, the intercept $(\hat{\alpha})$ of dashed lines 436 represents the natural logarithm of CSTV. 

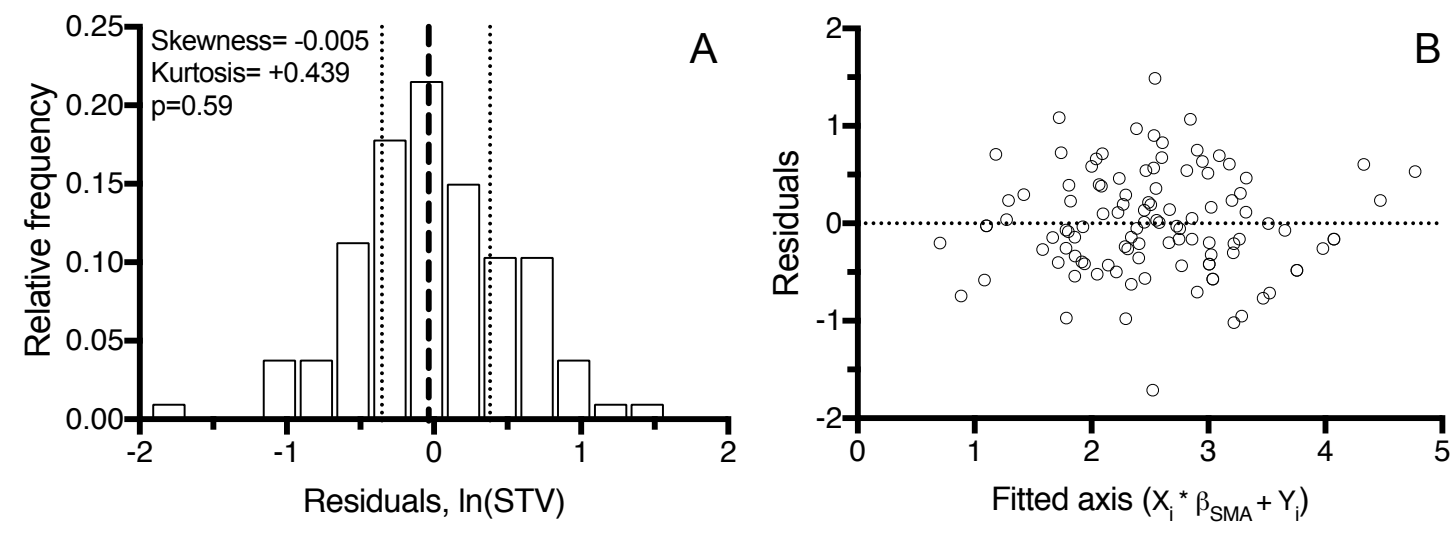

438

439 Fig. 3. Residual distribution for testing normality (A) and homoscedasticity (B) for the SMA regression 440 model of transformed variables applying the modified-ALCC methodology of Dataset \#1 (Fig. 2 B). The

441 Skewness and Kurtosis values indicate the level of asymmetry and bias of dataset. Vertical dotted lines 442 indicate percentiles 25, 50 (median) and 75 of distribution. Significance of the D'Agostino-Pearson 443 normality test is indicated with the p-value (D'Agostino et al., 1990). Homogeneity of variances of SMA 444 regression is tested visually against the fitted axis as described by Warton et al. (2006). 


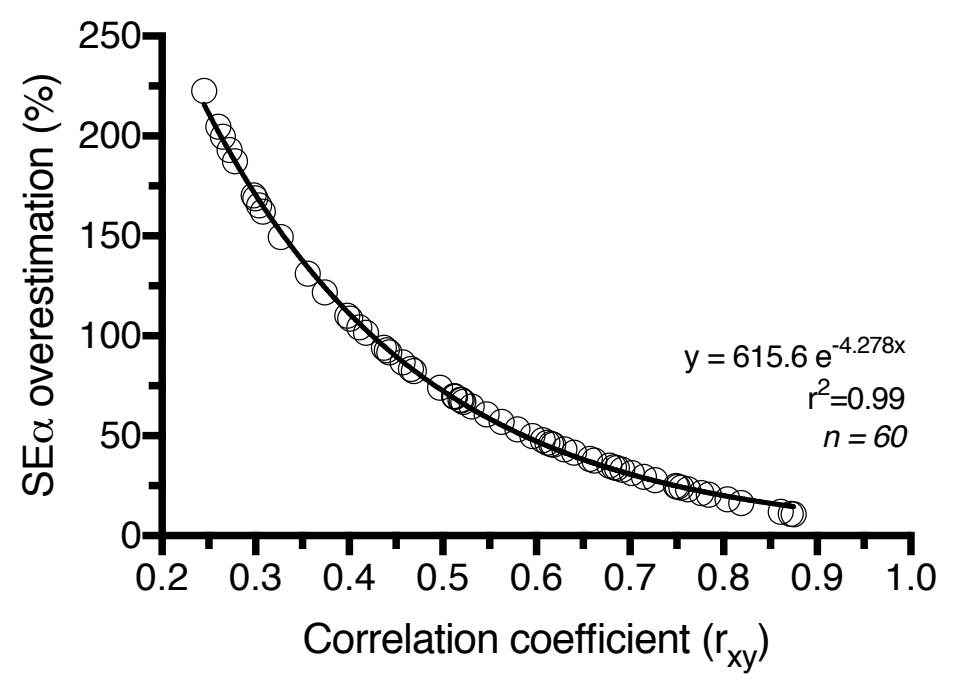

447 Fig. 4. Relationship between the correlation coefficient $\left(r_{x y}\right)$ of a dataset and the relative overestimation 448 of the standard error of the intercept (SE) using the original-ALCC method in comparison to the 449 modified-ALCC (Eqn. 14). The overestimation is related to the r-modification procedure that the original450 ALCC algorithm requires to estimate the bivariate equation of interest (standardized major axis). A total 451 of 60 datasets with different $r_{x y}$ were used (Dataset \#3). 

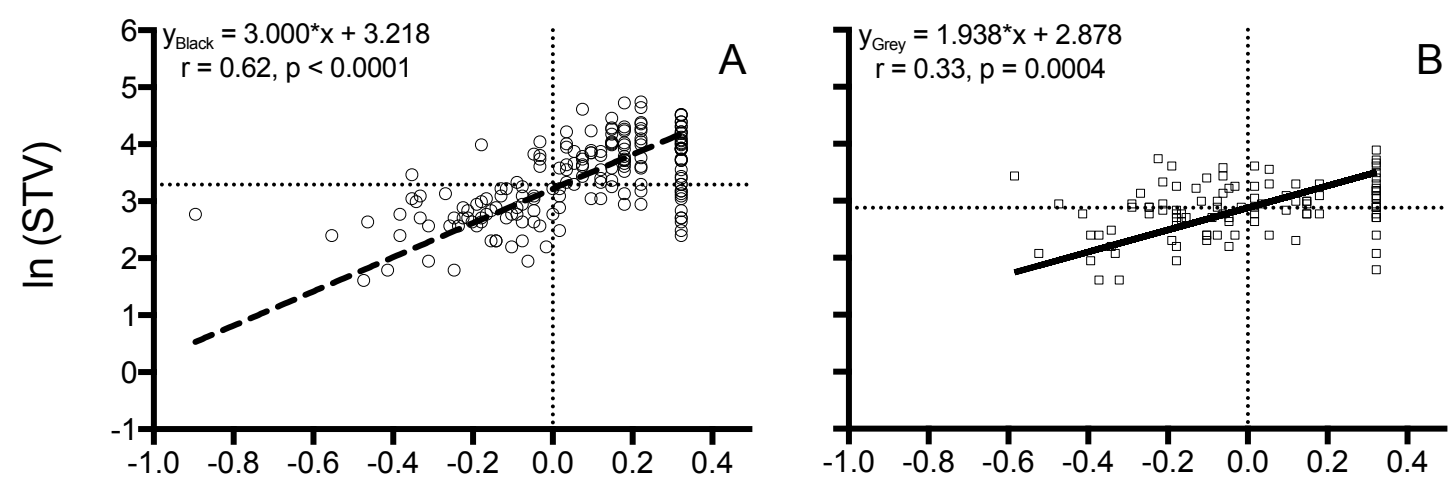

Arcsine $-\sqrt{ }\left(\mathrm{RY}_{90}\right)$
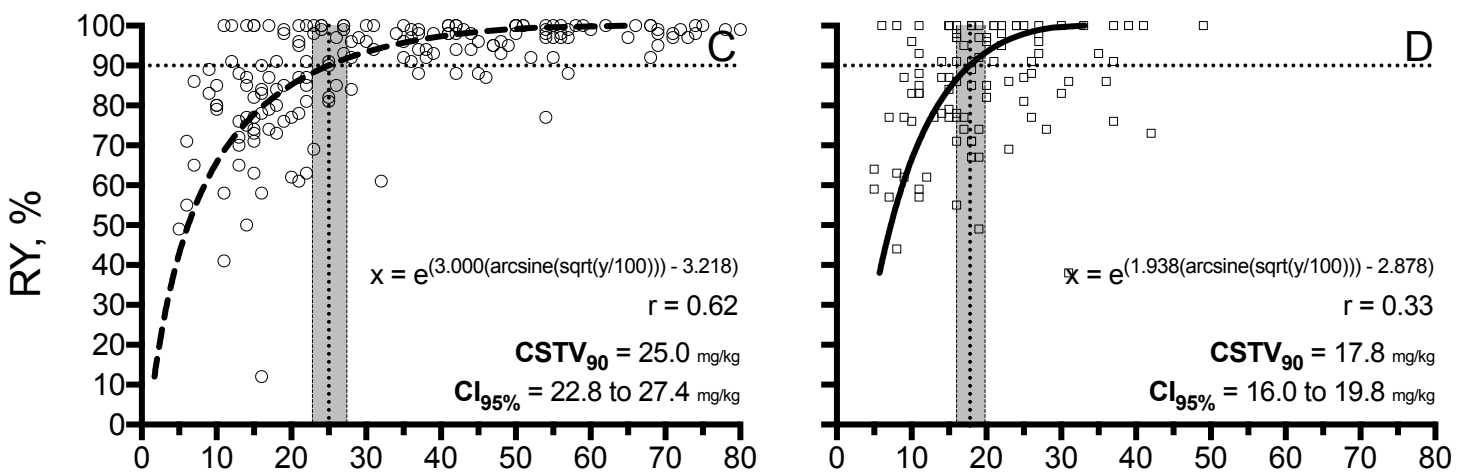

Colwell P $(0-10 \mathrm{~cm}), \mathrm{mg} / \mathrm{kg}$

454 Fig. 5. Relationships between wheat relative yield and soil Colwell $P$ concentration $(0-10 \mathrm{~cm}, \mathrm{mg} / \mathrm{kg})$ for

455 two soil types in Australia (Dataset \#2). Data was gathered from the BFDC Interrogator filtering by $P$ 456 response trials in cereal wheat under dryland conditions in Vertosol Black ( $A$ and $C, n=180)$ and Vertosol 457 Grey soils ( $B$ and $D, n=103$ ). A and $B$ show the bivariate linear regressions (standardized major axis) 458 between transformed variables, while $C$ and $D$ show the same relationships, back-transformed to the original units. Critical values (CSTV) and their confidence intervals ( $\mathrm{Cl}$, grey vertical strips) were estimated for $90 \%$ of RY with a $95 \%$ confidence level.

461

462

463

464 
466 Table 1. Comparison of confidence limits of critical soil test values (CSTV) estimates using the modified-

467 ALCC and the original-ALCC methods at two levels of confidence (95\% and $70 \%)$. Calculations were

468 made for soil Colwell-P at $0-10 \mathrm{~cm}(\mathrm{mg} / \mathrm{kg})$ at three levels of wheat relative yield (RY $80 \%, 90 \%$ and

469 95\%). Data was gathered from the BFDC Interrogator database (Dataset \#2).

\begin{tabular}{|c|c|c|c|c|c|c|c|}
\hline \multirow{3}{*}{$\begin{array}{c}\text { Vertosol Grey } \\
(n=103, r=0.33)\end{array}$} & \multirow{3}{*}{ CSTV } & \multicolumn{2}{|c|}{$80 \% R Y$} & \multicolumn{2}{|c|}{$90 \% R Y$} & \multicolumn{2}{|c|}{$95 \% R Y$} \\
\hline & & \multicolumn{2}{|c|}{13.5} & \multicolumn{2}{|c|}{17.8} & \multicolumn{2}{|c|}{21.4} \\
\hline & & Lower & Upper & Lower & Upper & Lower & Upper \\
\hline \multirow{2}{*}{ 95\% Confidence } & Modified & 11.9 & 15.3 & 16.0 & 19.8 & 19.1 & 24.1 \\
\hline & Original & 9.9 & 18.3 & 13.6 & 23.2 & 16.0 & 28.7 \\
\hline \multirow{2}{*}{ 70\% Confidence } & Modified & 12.7 & 14.4 & 16.8 & 18.8 & 20.1 & 22.8 \\
\hline & Original & 11.5 & 15.9 & 15.4 & 20.5 & 18.4 & 25.0 \\
\hline \multirow{2}{*}{$\begin{array}{c}\text { Vertosol Black } \\
(n=180, r=0.62)\end{array}$} & \multirow{2}{*}{ CSTV } & \multicolumn{2}{|c|}{16.3} & \multicolumn{2}{|c|}{25.0} & \multicolumn{2}{|c|}{33.3} \\
\hline & & Lower & Upper & Lower & Upper & Lower & Upper \\
\hline \multirow{2}{*}{ 95\% Confidence } & Modified & 14.5 & 18.4 & 22.8 & 27.4 & 30.5 & 36.4 \\
\hline & Original & 13.7 & 19.4 & 21.9 & 28.5 & 29.3 & 37.9 \\
\hline \multirow{2}{*}{ 70\% Confidence } & Modified & 15.3 & 17.3 & 23.8 & 26.2 & 31.8 & 34.9 \\
\hline & Original & 14.9 & 17.9 & 23.3 & 26.8 & 31.1 & 35.7 \\
\hline
\end{tabular}

470 